Conf $-9505105--13$ GA-A22051

\title{
FIRST DEMONSTRATION OF A TRAVELING WAVE ANTENNA IN A TOKAMAK AND RELEVANCE TO THE JFT-2M COMBLINE
}

\author{
by \\ D.A. PHELPS, C.P. MOELLER, H. IKEZI, \\ and S.C. CHIU
}

JUNE 1995 


\section{DISCLAIMER}

Portions of this document may be illegible in electronic image products. Images are produced from the best available original document. 


\title{
FIRST DEMONSTRATION OF A TRAVELING WAVE ANTENNA IN A TOKAMAK AND RELEVANCE TO THE JFT-2M COMBLINE
}

\author{
by \\ D.A. PHELPS, C.P. MOELLER, H. IKEZI, \\ and S.C. CHIU
}

This is a preprint of a paper presented at the 11th Topical Conference on Radio Frequency Power in Plasmas, May 17-19, 1995, Palm Springs, California, and to be printed in the Proceedings.

\author{
Work supported by \\ U.S. Department of Energy \\ Contract DE-AC03-89ER51114
}

\section{GENERAL ATOMICS PROJECT 3466 JUNE 1995}




\title{
First Demonstration of a Traveling Wave Antenna in a Tokamak and Relevance to the JFT-2M Combline
}

\author{
D.A. Phelps, C.P. Moeller, H. Ikezi, and S.C. Chiu
}

General Atomics, San Diego, California 92186

\begin{abstract}
First observations of a traveling wave antenna (TWA) in a tokamak are reported. For a fractional bandwidth $(\delta \omega / \omega)$ of about $20 \%$, TWA experiments using a four-element antenna in DIII-D showed less than $1 \%$ reflected power and excellent stability of the phase velocity of the launched wave - even during giant ELMs. The power radiated into the plasma varied from about $40 \%$ during ELM-free $\mathrm{H}$-mode to almost $90 \%$ during L-mode and ELMing H-mode. Theoretical modeling validated by these low power observations is used to predict the plasma load resistance of a high power combline being built for JFT-2M.
\end{abstract}

The technical challenge for fast wave current drive antennas in tokamaks is to maintain good plasma coupling, impedance matching, and array directivity during changing plasma conditions [1,2]. The traveling wave antenna (TWA) [2-7] is a new and promising way to meet this challenge. Like conventional arrays of actively driven elements [8,9], a TWA is a toroidal phased array of plasma facing elements in poloidal standing wave resonance. Unlike them, however, a TWA features filter-like passbands wherein power is coupled to one end element, propagated along the array via coupling between elements and terminated, or extracted, at the other end.

At the last RF Conference, the analysis and design of a combline for JFT-2M [4] and an externally tuned TWA for DIII-D [5] was reported. Using a dummy load to represent the plasma, experiments were presented on single pass operation $[4,5]$ and recirculation of power in a traveling wave resonator [5].

The new experiments reported herein were performed using a DIII-D four-element array described elsewhere [8]. As sketched in Fig. 1, a $30 \Omega$ vacuum coaxial line connects each element through a DIII-D feedthrough 


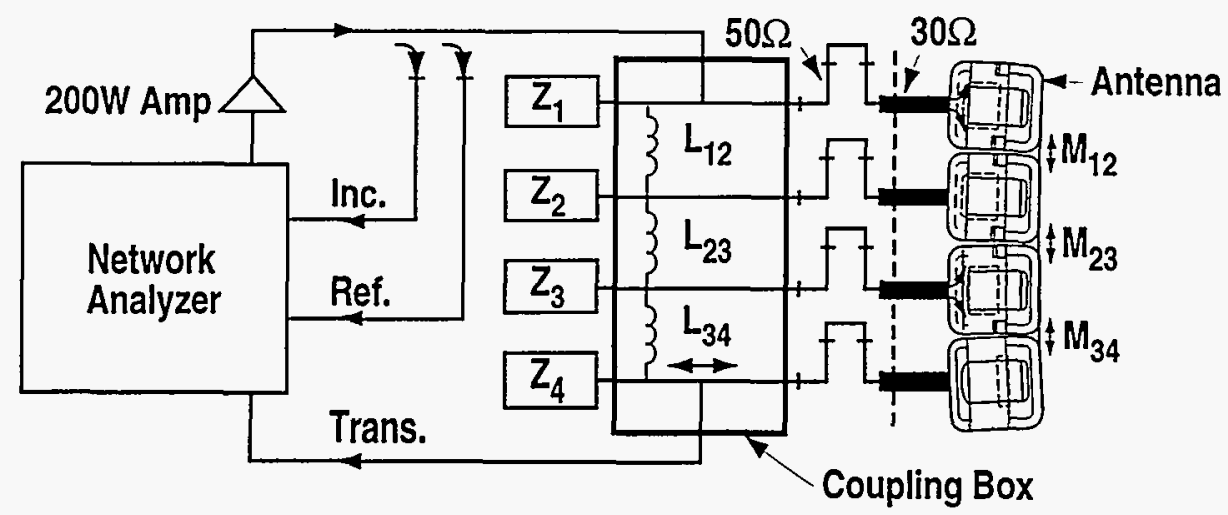

FIGURE 1. A schematic drawing of the if circuit for externally coupled TWAs.

to $50 \Omega$ cable and thence to a copper rod conductor in the "coupling box." The other end of each rod terminates in an open or shorted cable of desired length. This forms a four-element TWA. Coupling inductors were added to change the bandwidth of this TWA.

Using a network analyzer, the TWA reflection and transmission coefficients in Fig. 2 were measured. At the optimum bandwidth of $\delta \omega / \omega=18 \%$, taken at the $3 \mathrm{~dB}$ points in Fig. 2(a), good repeatability was observed under similar plasma conditions. The transmission amplitudes without plasma and with $\mathrm{L}$-mode plasma are consistent with the theoretical waveforms to be discussed later. Figure 2(b) demonstrates an acceptable few degrees per element phase shift. Figure 2 (c) exhibits $\lesssim-20 \mathrm{~dB}$ reflected power within the plateau even for a change from vacuum to $\mathrm{L}$-mode plasma loading. At the widest bandwidth of $\delta \omega / \omega=30 \%$ the attenuation was too weak. At the minimum of $\delta \omega / \omega=3 \%$ the attenuation was too strong - around $20 \mathrm{~dB}$ during the L-mode and ELMs. There was also significant frequency upshift in the passband.

Significant changes in plasma conditions are observed in the DIII-D plasma diagnostics shown in Fig. 3 (a) through (d). These changes affect the transmission through the $18 \%$ bandwidth TWA as shown in Fig. 3(e). Note around the $\mathrm{L}-\mathrm{H}$ transition and into the ELMing $\mathrm{H}$-mode that the
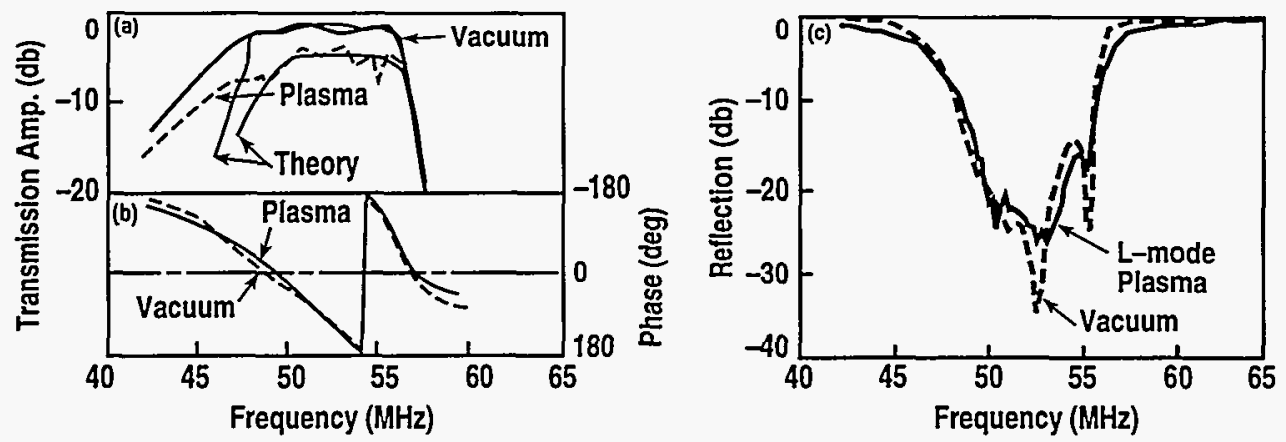

FIGURE 2. (a) Transmission amplitude and (b) phase, and (c) reflection amplitude $\left(B_{\mathrm{T}}=2.1 \mathrm{~T} ; \mathrm{I}_{\mathrm{p}}=700 \mathrm{kA} ; \mathrm{n}_{\mathrm{e} 0}=3\right.$ to $\left.5 \times 10^{19} \mathrm{~m}^{-3}\right)$. 

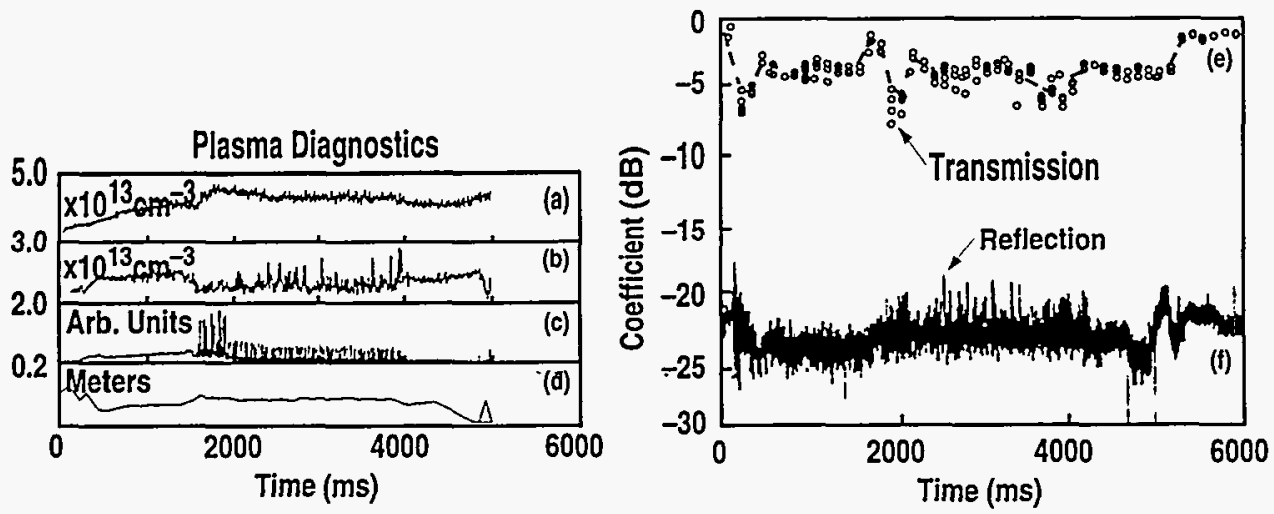

FIGURE 3. Time resolved comparison of (a) the central and (b) the edge density variations in DIII-D; (c) $D_{\alpha}$ emission from the divertor; (d) the gap between the plasma and the antenna; (e) the transmitted and ( $f$ ) the reflected power $\left(B_{\mathrm{T}}=2.1 \mathrm{~T}, \mathrm{I}_{\mathrm{p}}=700 \mathrm{kA}\right)$.

attenuation first decreases from 4 to $6 \mathrm{~dB}$ to 1 to $2 \mathrm{~dB}$ and then increases again to 4 to $8 \mathrm{~dB}$. This represents a range of $40 \%$ to $90 \%$ in power coupling efficiency to the plasma. In spite of these changes in coupled power, the reflected power in Fig. $3(\mathrm{f})$ is always $<-20 \mathrm{~dB}$ (or $1 \%$ as measured at a fixed frequency of $53 \mathrm{MHz}$ ). This resilience to plasma variations is also seen in the phase of the reflection and transmission coefficients [6,7], and thereby the phase velocity of the TWA.

A coupled TWA model described elsewhere [6,7] assumes a circuit with each element in Fig. 1 being a pair of poloidally stacked magnetic loops connected by $30 \Omega$ transmission line to external shorts and/or coupling inductors. The good agreement between the experimental and theoretical waveforms shown in Fig. 2(a) validates this model. Using an $18 \%$ bandwidth TWA, a $30 \Omega$ characteristic input impedance, and the attenuation data in Fig. 3 , load resistances of $2.3 \Omega$ during the L-mode and $1.2 \Omega$ during the ELM-free $\mathrm{H}$-mode (to within $\pm 25 \%$ ) are predicted for the TWA in DIII-D.

Next we use our validated theory to predict the plasma loading of the high power combline being built for JFT-2M. A mock-up of this combline is shown and described elsewhere [4]. In brief, fifteen $2.5 \mathrm{~cm}$-wide elements are located on $5.6 \mathrm{~cm}$ centers. A $75 \mathrm{deg}$ phase between elements yields the desired index of refraction of 5.5 [11]. The transmission and reflection coefficient waveforms in Fig. 4 were achieved during cold tests by optimizing the tap points and terminating capacitance of the end elements only. We measured a characteristic impedance of $Z_{0}=67 \Omega, v=0.7 \mathrm{c}$, and a $43 \%$ bandwidth between the sharp reflected power cutoffs.

Microwave absorber in front of the TWA caused about $9 \mathrm{~dB}$ attenuation (i.e., $0.6 \mathrm{~dB}$ per element) near 75 to $90 \mathrm{deg}$ phase shifts per element in Fig. 4. We compared this result with the theory validated above [6] and a theory that treats the antenna and coax as coupled lossy transmission line [10] with only mutual coupling. The average resistance predicted by the two theories is $2 \Omega$ (to within 10\%). Up to $1.6 \mathrm{~dB}$ (or 5 to $6 \Omega$ per element) was also 

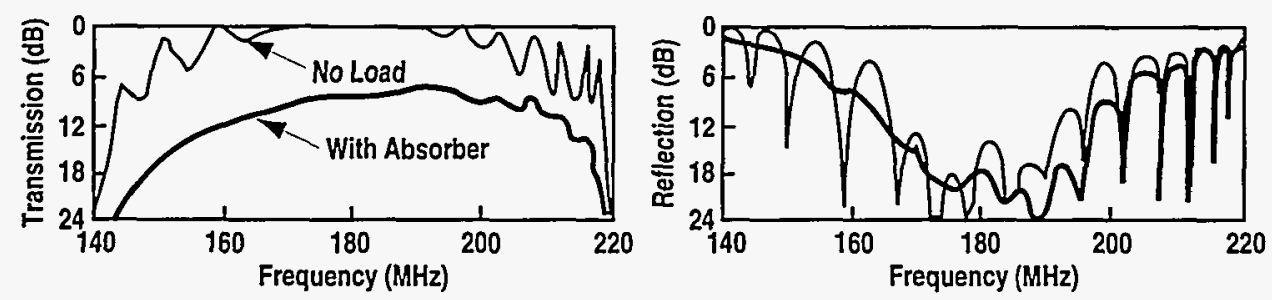

FIGURE 4. Measurements of transmission and reflection coefficient waveforms with and without absorber added to front of Faraday shield.

observed. For comparison, the resistance of a four-element JFT-2M antenna [11] with a similar index of refraction is 1.7 to $5.4 \Omega$ at $67 \Omega$. Good combline performance under JFT-2M plasma loading conditions is thus anticipated.

In conclusion, for a $15 \%$ to $20 \%$ bandwidth, the TWA match and phase velocity are resilient to plasma variations in DIII-D and $40 \%$ to $90 \%$ coupled power is observed during the L-mode and grassy Elming $\mathrm{H}$-mode (of interest to ITER and TPX). These observations combine with theory and accepted high voltage rf engineering $[8,9]$ to validate high power TWAs. In particular, they predict over $90 \%$ coupling efficiency even during ELMing $\mathrm{H}$-mode for ten or more element TWAs in DIII-D, as well as the fifteen-strap combline for JFT-2M. For four-element arrays in DIII-D, a traveling wave resonator is being evaluated $[5,6]$.

This is a report of work sponsored by the U.S. Department of Energy under Contract No. DE-AC03-89ER51114.

\section{REFERENCES}

[1] D.B. Remsen et al., in Proceedings of the 15th Symposium on Fusion Engineering, Piscataway, 1993, p. 1088.

[2] D.A. Phelps et al., Bull. Am. Phys. Soc. 38, 2069 (1993).

[3] S.C. Chiu et al., Nucl. Fusion 24, 717 (1984).

[4] C.P. Moeller et al., in Proceedings of the 10th Topical Conference on Radio Frequency Power in Plasmas, Boston, 1993, p. 323.

[5] D.A. Phelps et al., ibid., p. 339.

[6] H. Ikezi, General Atomics, personal correspondence, 1995.

[7] D.A. Phelps et al., Bull. Am. Phys. Soc. 39, 1649 (1994).

[8] F.W. Baity et al., in Proceedings of the 10th Topical Conference on Radio Frequency Power in Plasmas, Boston, 1993, p. 343.

[9] A. Kaye et al., Fusion Technol. 13, 203 (1987).

[10] R.H. Goulding et al., Fusion Eng. and Design 24, 104 (1994).

[11] M. Saigusa et al., Nucl. Fusion 33, 421 (1993). 